\title{
Cluster or periodic, static or dynamic - the challenge of calculating the $g$ tensor of the solid-state glycine radical
}

\author{
Ewald Pauwels, ${ }^{* a b}$ James Asher, ${ }^{c}$ Martin Kaupp ${ }^{c}$ and Michel Waroquier ${ }^{a b}$ \\ Received 6th May 2011, Accepted 31st August 2011 \\ DOI: $10.1039 / \mathrm{c} 1 \mathrm{cp} 21452 \mathrm{~g}$
}

The calculation of the $g$ tensor of the main ${ }^{+} \mathrm{NH}_{3}{ }^{\cdot} \mathrm{CH}-\mathrm{COO}^{-}$radiation-induced radical in solid-state $\alpha$-glycine presents a real challenge to computational methods. Density functional calculations of this spectroscopic property struggle with its small anisotropy and the zwitterionic nature of the amino acids in the crystal of this seemingly simple system. Here, several factors influencing the calculated $g$ tensor are examined by comparing with experimental data. The extent of the molecular environment is varied in both a cluster and a periodic approach and dynamic calculations are performed to account for temperature effects. The latter does not necessarily lead to a better agreement with experiment than a static calculation. Application of a periodic approach is straightforward, but an all-electron scheme clearly is favorable. In a cluster approach, the selected basis set and density functional are of less importance, provided a hybrid functional is used to prevent cluster boundary effects. The applied spin-orbit coupling operators and proper treatment of the gauge origin of the magnetic vector potential also seem to be less critical than in other, similar molecular systems. But a careful selection of the cluster size proves to be essential for this glycine radical system. The calculated $g$ tensor varies significantly with increasing cluster size, yielding only a good agreement with experiment when 5-7 glycine molecules in the immediate environment of the central glycine radical are incorporated. Further expansion of the cluster size can even lead to an essentially incorrect description of the radical in the condensed phase, indicating that bigger clusters can become unbalanced.

\section{Introduction}

The study of paramagnetic centers in condensed-phase biomolecules has attracted considerable interest in recent years. In proteins, for instance, radicals are often at the heart of the biochemical catalytic process. ${ }^{1}$ In DNA, radicals are mostly associated with radiation damage ${ }^{2}$ and several studies have been devoted to these paramagnetic species in model systems (e.g. ref. 3-9).

Electron paramagnetic resonance (EPR) spectroscopy (or derived techniques) can give a unique insight in both the electronic and structural properties of a radical through its main spectroscopic parameters: the hyperfine coupling tensors and the $g$ tensor. EPR experiments are increasingly complemented by $a b$ initio calculations based on density functional theory (DFT). These methods allow the explicit optimization of a radical structure and the calculation of its EPR parameters, ${ }^{10}$ which in turn can serve to verify and validate experimental models and assumptions.

${ }^{a}$ Center for Molecular Modeling, Ghent University, Technologiepark

903,B-9052 Zwijnaarde, Belgium.E-mail: ewald.pauwels@UGent.be

${ }^{b}$ QCMM-alliance Ghent-Brussels, Belgium

${ }^{c}$ Technische Universität Berlin, Institut für Chemie, Theoretische

Chemie, Sekr. C 7, Straße des 17. Juni 135, 10623 Berlin, Germany
A recurring problem in simulations of biomolecular radicals in the condensed phase is the treatment of the molecular environment. The environment influences the electronic as well as the structural properties of a radical and therefore needs to be accounted for appropriately. Several approaches have been adopted in the literature, the most rudimentary being just to neglect the environment (e.g. ref. 11). This is improved in a cluster approach, where part of the molecular environment of the radical is explicitly considered in the calculations (e.g. ref. 12). An alternative improvement can be achieved in a periodic approach, in which a unit cell containing the radical and some of the molecules surrounding it are subject to periodic boundary conditions (e.g. ref. 13). Owing to its cost-effective incorporation of even long-range intermolecular interactions, this method is perhaps the most suitable to date to examine radicals in the condensed phase, limited only by the size of the unit cell and the associated computational cost. Another important factor in biomolecular simulations is temperature. Whereas calculations are most easily performed on single, static structures (e.g. the optimized structure), state-of-the-art simulations take into account the dynamic nature of the system at a finite temperature. This can be achieved by sufficiently sampling the potential energy surface of the system, often with the aid of molecular dynamics (MD) (e.g. ref. 14 and 15). 
In previous studies, ${ }^{16,17}$ the importance of the molecular environment and temperature in simulations was evaluated for the main ${ }^{+} \mathrm{NH}_{3}-{ }^{\bullet} \mathrm{CH}-\mathrm{COO}^{-}$radiation-induced radical in solid-state $\alpha$-glycine and its associated hyperfine coupling tensors. This crystalline amino acid is a benchmark system to study the damaging effects of radiation on proteins. ${ }^{18}$ It has attracted experimental ${ }^{19-25}$ as well as theoretical ${ }^{16,17,26-28}$ attention. Several approaches to simulate the molecular environment were evaluated $^{16}$ and it was established that at least a cluster or a periodic approach is crucial for a correct description of the structure and conformation of the glycine radical. But also in the subsequent calculation of spectroscopic properties, the chosen approach can have a non-negligible influence. The hyperfine coupling tensors, and especially their principal directions, significantly improved when the molecular environment was included in the computational scheme. ${ }^{16}$ Temperature-accounted for by MD simulations - only moderately affected the hyperfine coupling tensors of the glycine radical, but can have a marked effect on the isotropic couplings. ${ }^{17}$

So far, one theoretical study is available that reports the calculated $g$ tensor for the solid-state glycine radical system. ${ }^{28}$ These researchers relied on a hybrid approach to determine this spectroscopic property: the structure was determined in a periodic code, after which EPR properties were calculated for a cluster that was cut out of the periodic structure. However, no analysis was made of the $g$-tensor principal directions and, unfortunately, comparison was made with experimental data ${ }^{20}$ that were misinterpreted (vide infra). Other computational studies have thoroughly examined the $g$ tensor of similar radicals in glycine-derivatives. ${ }^{29,30}$ In these systems, the very small $g$-anisotropy, delocalized spin density and interaction with the molecular environment proved particularly challenging in the calculation of the $g$ tensor, necessitating primarily a proper choice for the spin-orbit operators and an appropriate treatment of the gauge of the external magnetic vector potential.

In this work, we complete our theoretical study of the main radiation-induced ${ }^{+} \mathrm{NH}_{3}-{ }^{\bullet} \mathrm{CH}-\mathrm{COO}^{-}$radical in solid-state $\alpha$-glycine and focus on the principal values and directions of its $g$ tensor. We examine to what extent this spectroscopic property is affected by the chosen computational scheme and perform periodic as well as cluster calculations to account for the molecular environment. In the cluster approach specifically, the effects of basis set, density functional and type of implementation are evaluated. In addition, we thoroughly study the cluster size effect and find that it can easily lead to misinterpretation. Finally, the effect of a finite temperature on the $g$ tensor is examined by relying on molecular dynamics simulations.

\section{Computational details}

The structure and dynamics of the main glycine radical were already explored in a previous work ${ }^{17}$ and the obtained structures and trajectories are reused in the present work. The optimized structure for the glycine radical was obtained by performing periodic calculations on a supercell, constructed by doubling the crystallographic glycine unit cell $^{31}$ in the $\langle a\rangle$ and $\langle c\rangle$ directions. One of the 16 molecules in the supercell was transformed into a radical by removing one of the hydrogens on $\mathrm{C}_{\alpha}$. Subsequent optimization under periodic boundary

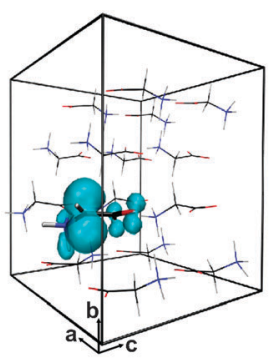

(a)

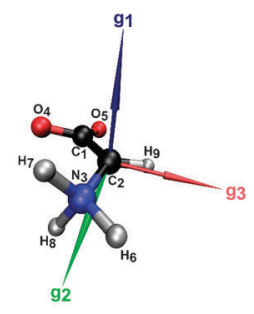

(b)

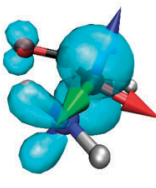

(c)
Fig. 1 (a) The glycine radical within the periodic supercell (optimized structure from ref. 17). Blue contours illustrate the spin density of the $\mathrm{CP} 2 \mathrm{~K}$ calculation (isovalue 0.0035 ). (b) Atom numbering scheme and orientation of the experimental $g$-tensor principal directions $\left(g_{1}-g_{3}\right)$. (c) Spin density and corresponding principal directions of the CSGT-VEFF $g$-tensor calculation of CP2K.

conditions was performed with the CPMD software, ${ }^{32}$ using the BP86 density functional ${ }^{33,34}$ and a plane wave basis set (cutoff 25.0 Ry) and ultra soft pseudopotentials of the Vanderbilt type. $^{35}$ The optimized structure is shown in Fig. 1(a). Atomic charges were derived from the electrostatic potential for this structure. ${ }^{36}$ A trajectory containing 400 snapshots (6 fs per snapshot) was taken from a 2.4 ps Car-Parrinello ${ }^{37}$ molecular dynamics simulation adopting the same level of theory. The MD time-step was 5 a.u. (0.12 fs) and the fictitious electronic mass was set to 400 a.u. Nosé-Hoover thermostats ${ }^{38}$ were applied to ensure an average temperature of $300 \mathrm{~K}$.

For the optimized structure, several computational schemes were used to calculate the $g$ (and hyperfine coupling) tensors. Periodic calculations were performed on the optimized periodic supercell using both the $\mathrm{CPMD}^{32}$ and $\mathrm{CP} 2 \mathrm{~K}^{39}$ software packages. In the former, Troullier-Martins norm-conserving pseudopotentials ${ }^{40}$ were used with a cutoff value of $100.0 \mathrm{Ry}$. In the latter, the Gaussian and augmented plane wave (GAPW) method ${ }^{41}$ was applied. In this scheme, the total density is described by a smooth, extended part that is represented by plane waves (density cutoff $200 \mathrm{Ry}$ ) and parts localized close to the nuclei are expanded in periodic Gaussian functions (all-electron TZVP basis ${ }^{42}$ ). The PBE functional ${ }^{43}$ was used throughout all periodic calculations. Cluster calculations of the EPR properties were performed with Gaussian03, ${ }^{44}$ using the B3LYP functional ${ }^{45}$ in combination with a TZVP basis set, ${ }^{42}$ unless specified otherwise. In addition, several $g$-tensor calculations were performed with the MAG/ReSpect code, ${ }^{46}$ relying on converged Kohn-Sham wave functions that were determined with Gaussian03.

The way in which the $g$-tensor calculation is implemented differs crucially between CPMD, CP2K, Gaussian03 and $\mathrm{MAG} / \mathrm{ReSpect}$. All rely on a second-order perturbation treatment of the Breit-Pauli Hamiltonian (see e.g. ref. 47), but crucially differ in their approximation of the spin-orbit coupling contribution to the $g$ tensor:

$$
\Delta g_{\mathrm{SO}}=\frac{g_{\mathrm{e}} \alpha^{2}}{2 S}\left[\sum_{n} \frac{\left\langle\Psi_{0}^{(0)}\left|\hat{H}_{\mathrm{SO}}\right| \Psi_{n}^{(0)}\right\rangle\left\langle\Psi_{n}^{(0)}\left|\hat{H}_{\mathrm{OZ}}\right| \Psi_{0}^{(0)}\right\rangle}{E_{0}^{(0)}-E_{n}^{(0)}}\right]
$$

where $g_{\mathrm{e}}$ is the free-electron $g$ value, $\alpha$ the fine structure constant, $S$ the total electron spin, $\Psi_{0}^{(0)}$ and $\Psi_{n}^{(0)}$ the unperturbed wavefunctions of the ground state and the $n$-th excited 
state, respectively, with corresponding eigenenergies $E_{0}^{(0)}$ and $E_{n}^{(0)}$. $\hat{H}_{\mathrm{OZ}}$ represents the orbital Zeeman interaction arising from the external magnetic field, but it is especially the treatment of the spin-orbit operator $\hat{H}_{\text {SO }}$ that differs between the mentioned implementations:

$$
\begin{aligned}
\hat{H}_{\mathrm{SO}}=\sum_{i} & \mathbf{s}_{i} \cdot\left\lfloor\sum_{N} Z_{N} \frac{\left(\mathbf{r}_{i}-\mathbf{R}_{N}\right)}{\left|\mathbf{r}_{i}-\mathbf{R}_{N}\right|^{3}} \times \mathbf{p}_{i}-\sum_{j \neq i} \frac{\left(\mathbf{r}_{i}-\mathbf{r}_{j}\right)}{\left|\mathbf{r}_{i}-\mathbf{r}_{j}\right|^{3}}\right. \\
& \left.\times \mathbf{p}_{i}-2 \sum_{j \neq i} \frac{\left(\mathbf{r}_{j}-\mathbf{r}_{i}\right)}{\left|\mathbf{r}_{j}-\mathbf{r}_{i}\right|^{3}} \times \mathbf{p}_{j}\right\rfloor
\end{aligned}
$$

with $\mathbf{s}_{i}$ the spin operator for electron $i, \mathbf{p}_{i}$ the electron momentum, $Z_{N}$ the charge of nucleus $N$ and $\mathbf{r}_{i}, \mathbf{R}_{N}$ are electron and nuclear position vectors, respectively. In Gaussian, following the implementation by Neese, ${ }^{48}$ this operator is approximated by introducing semi-empirically chosen effective nuclear charges that were derived by Koseki et al. ${ }^{49}$ (shorthand KOSEKI):

$$
\hat{H}_{\mathrm{SO}}=\sum_{i} \mathbf{s}_{i} \cdot \sum_{N} Z_{N}^{\text {eff }} \frac{\left(\mathbf{r}_{i}-\mathbf{R}_{N}\right)}{\left|\mathbf{r}_{i}-\mathbf{R}_{N}\right|^{3}} \times \mathbf{p}_{i}
$$

Gauge-including atomic orbitals $\left(\right.$ GIAOs $^{50,51}$ ) are used to handle the gauge of the external magnetic vector potential. In the $\mathrm{CPMD}^{52}$ and $\mathrm{CP} 2 \mathrm{~K}^{53}$ implementations, a periodic variant of the Schreckenbach and Ziegler approximation ${ }^{54}$ is essentially followed (referred to with the shorthand VEFF):

$$
\hat{H}_{\mathrm{SO}}=\sum_{i} \mathbf{s}_{i} \cdot\left(\bar{\nabla} V_{\mathrm{eff}}^{\mathrm{KS}} \times \mathbf{p}_{i}\right)
$$

in which the gradient of the effective (Kohn-Sham) potential is used, augmented with an approximate treatment of the spin-other-orbit contribution (the last term in eqn (2)) due to Pickard and Mauri. ${ }^{55}$ In addition, the "continuous set of gauge transformation' method (CSGT) by Keith and Bader ${ }^{56}$ is applied. Finally, in MAG/ReSpect, various treatments of the spin-orbit integrals of eqn (1) are possible, ${ }^{57}$ ranging from the simple KOSEKI approximation to a full treatment of all one- and two-electron integrals (FULL). It also features the atomic meanfield approximation (AMFI), ${ }^{58,59}$ in which all multi-center one- and two-electron integrals are neglected, reducing eqn (2) to an integration over effective atomic spin-orbit operators, which is particularly interesting from a computational point of view when dealing with larger molecular systems. The gauge problem can be addressed with a common gauge approximation (CG) as well as with GIAOs.

\section{Results and discussion}

\section{Considerations on experimental data}

Two experimental studies report on the $g$ tensor of the glycine radical (Table 1). In 1966, Collins and Whiffen ${ }^{21}$ determined a complete $g$ tensor using EPR and ENDOR (Electron Nuclear Double Resonance) in the X- and Q-band. More recently, Brustolon et al. ${ }^{20}$ used high-frequency EPR but only measured the non-diagonal $g$ value along the crystallographic $\langle c\rangle$ axis. Their value (2.00394) is consistent with the corresponding projection using the 1966 data (2.00388), and provides validation for that complete $g$ tensor, even though its accuracy is rather limited: an error margin of $100 \mathrm{ppm}$.
Table 1 Overview of experimental $g$ tensor data. $g_{\text {iso }}$ and $g_{\text {aniso }}$ refer to the isotropic (average) and anisotropic $g$ values, respectively. The

\begin{tabular}{|c|c|c|c|c|c|c|c|}
\hline & \multirow[b]{2}{*}{$g_{\text {iso }}$} & \multirow[b]{2}{*}{$g_{\text {aniso }}$} & \multirow[b]{2}{*}{ Label } & \multicolumn{3}{|c|}{ Principal directions } & \multirow[b]{2}{*}{$B / /\langle c\rangle$} \\
\hline & & & & $\left\langle a^{*}\right\rangle$ & $\langle b\rangle$ & $\langle c\rangle$ & \\
\hline \multirow[t]{3}{*}{ Ref. 21} & 2.0036 & 2.0032 & $g_{1}$ & 0.490 & 0.842 & 0.227 & 2.00388 \\
\hline & & 2.0035 & $g_{2}$ & 0.844 & -0.392 & -0.367 & \\
\hline & & 2.0042 & $g_{3}$ & 0.220 & -0.371 & 0.902 & \\
\hline Ref. 20 & & & & & & & 2.00394 \\
\hline
\end{tabular}
principal directions are given as cosines with respect to the crystallographic axes. The last column lists the non-diagonal $g$ value, as measured for the magnetic field along the $\langle c\rangle$ axis

As is characteristic for this kind of amino-acid radicals, ${ }^{60}$ the $g$ tensor has a small anisotropy and the principal direction corresponding to the minimum $g$ value $\left(g_{1}\right)$ is parallel to the p-like lone electron orbital of this $\pi$-type radical. The other principal directions corresponding to the intermediate and maximum $g$ values ( $g_{2}$ and $g_{3}$ ) are in the plane of the radical, with the $g_{2}$-direction roughly along the $\mathrm{C}_{2}-\mathrm{N}_{3}$ bond. This is clear from Fig. 1(b), where the experimental principal directions are superimposed on the optimized glycine radical structure. The $g_{1}$ value of 2.0032 is quite large for this type of amino-acid radicals. As alanine ${ }^{60}$ or other glycine radicals ${ }^{61}$ yield typical values close to the free electron value, possibly a typographical error has been made in the original publication. ${ }^{21}$ Simulations of the EPR and ENDOR angular variations using both the original and corrected $g$ tensor were similar to those reported, but none could be favored over the other.

\section{Periodic calculations}

Once a structure has been determined within a periodic scheme, it is most straightforward to also calculate the EPR properties within that framework. Only a few packages allow the calculation of the $g$ tensor within a periodic approach, two of which are applied here. Table 2(a) lists the results of the CPMD and $\mathrm{CP} 2 \mathrm{~K}$ calculations, both relying on the CSGT-VEFF scheme (see Computational details). Both packages succeed in reproducing the small anisotropy of the $g$ tensor, but the principal values can easily differ by $500-1000$ ppm with respect to experiment. This is somewhat above the typical accuracy of computational methods. ${ }^{48}$ Most likely, this is due to the use of the Pickard-Mauri spin-other-orbit operator, ${ }^{55}$ which has been noted to yield an underestimate of this contribution. ${ }^{47}$ The CP2K calculation seems to outperform CPMD for the $g_{2}$ and $g_{3}$ values, which are more strongly overestimated in the latter. Both periodic schemes align the $g_{1}$ principal direction along the lone electron orbital, which bears the majority of spin density at the $\mathrm{C}_{2}$ radical center (Fig. 1(c)). Hence, the deviation (in degrees) between the calculated and experimental principal directions is below $20^{\circ}$, which can be considered satisfactory. ${ }^{16}$ The $g_{2}$ and $g_{3}$ principal directions, on the other hand, are only well aligned in the $\mathrm{CP} 2 \mathrm{~K}$ calculation. These directions are clearly most sensitive to the small changes in the spin density associated with the different computational schemes. The neglect of explicit core electrons due to the use of pseudopotentials in CPMD seems to have a pronounced effect on the $g_{2}$ and $g_{3}$ principal values and directions. Conclusively, an all-electron periodic scheme (as with GAPW 
Table 2 Overview of $g$-tensor calculations in a periodic and cluster approach, using an optimized structure determined with CPMD and a BP86 functional (see Computational details). The angles (in degrees) indicate the deviation between corresponding calculated and experimental principal directions. The B3LYP functional and TZVP basis set were used for all calculations in (b), except the GIAO-FULL case where the smaller SV set was used for all molecules surrounding the radical in the cluster. The $\mathrm{Cl}$ atom was used as a common gauge in the CG-AMFI calculation

\begin{tabular}{|c|c|c|c|c|c|}
\hline \multirow[b]{2}{*}{ Experiment } & \multirow[b]{2}{*}{$g_{\text {iso }}$} & \multirow[b]{2}{*}{$g_{\text {aniso }}$} & \multicolumn{3}{|c|}{ Direction cosines } \\
\hline & & & $\left\langle a^{*}\right\rangle$ & $\langle b\rangle$ & $\langle c\rangle$ \\
\hline & \multirow[t]{4}{*}{2.0036} & 2.0032 & 0.490 & 0.842 & 0.227 \\
\hline & & 2.0035 & 0.844 & -0.392 & -0.367 \\
\hline & & 2.0042 & 0.220 & -0.371 & 0.902 \\
\hline & & $g_{\text {iso }}$ & \multicolumn{2}{|c|}{$g_{\text {aniso }}$} & Angle \\
\hline \multicolumn{6}{|c|}{ (a) Periodic method } \\
\hline CSGT-VEF & & \multirow[t]{3}{*}{2.0042} & \multicolumn{2}{|c|}{2.0022} & 13 \\
\hline \multirow{2}{*}{\multicolumn{2}{|c|}{ CPMD }} & & \multirow{2}{*}{\multicolumn{2}{|c|}{$\begin{array}{l}2.0046 \\
2.0051\end{array}$}} & 55 \\
\hline & & & & & 55 \\
\hline \multicolumn{2}{|c|}{ CSGT-VEFF } & \multirow[t]{3}{*}{2.0038} & \multicolumn{2}{|c|}{2.0022} & 17 \\
\hline \multirow{2}{*}{\multicolumn{2}{|c|}{$\mathrm{CP} 2 \mathrm{~K}$}} & & \multirow{2}{*}{\multicolumn{2}{|c|}{$\begin{array}{l}2.0043 \\
2.0047\end{array}$}} & 16 \\
\hline & & & & & 14 \\
\hline \multicolumn{6}{|c|}{ (b) Cluster method } \\
\hline \multicolumn{2}{|c|}{ GIAO-KOSEKI } & 2.0036 & \multicolumn{2}{|c|}{2.0022} & 18 \\
\hline \multicolumn{2}{|l|}{ TZVP } & & \multicolumn{2}{|c|}{2.0040} & 16 \\
\hline \multicolumn{2}{|c|}{$\begin{array}{l}\text { Gaussian03 } \\
\text { GIAO-FULL }\end{array}$} & & \multicolumn{2}{|c|}{2.0045} & 13 \\
\hline \multirow{2}{*}{\multicolumn{2}{|c|}{$\begin{array}{l}\text { GIAO-FULL } \\
\text { TZVP-SV }\end{array}$}} & 2.0035 & \multirow{2}{*}{\multicolumn{2}{|c|}{$\begin{array}{l}2.0023 \\
2.0039\end{array}$}} & 17 \\
\hline & & & & & 13 \\
\hline \multicolumn{2}{|c|}{ MAG/ReSpect } & & \multicolumn{2}{|c|}{2.0043} & 10 \\
\hline \multicolumn{2}{|l|}{ CG-AMFI } & 2.0035 & \multicolumn{2}{|c|}{2.0023} & 18 \\
\hline \multicolumn{2}{|c|}{ TZVP } & & \multicolumn{2}{|c|}{2.0039} & 15 \\
\hline \multicolumn{2}{|c|}{ MAG/ReSpect } & & \multicolumn{2}{|c|}{2.0043} & 11 \\
\hline
\end{tabular}

in $\mathrm{CP} 2 \mathrm{~K}$ ) for the calculation of the $g$ tensor yields a better agreement with experiment.

\section{Cluster calculations-methodological effects}

Several cluster models can be constructed on the basis of the optimized periodic structure. Including only the nearest intermolecular interactions, a cluster model is readily obtained containing the radical and six glycine molecules that are hydrogen bound to it. This 6-Cluster was used previously and satisfactorily reproduced experimental hyperfine coupling tensors. ${ }^{16,17}$

In Table 2(b), comparison is made of the $g$ tensors calculated with several non-periodic approaches using this particular cluster model and a B3LYP ${ }^{45}$ density functional. Despite the methodological differences between these methods (see Computational details), all yield quite comparable results. Overall, the agreement of the cluster calculations with the experimental $g$ tensor is rather good and even slightly better than the CP2K results. The GIAO-FULL and CG-AMFI approaches of $\mathrm{MAG} / \mathrm{ReSpect}$ perform better in the prediction of the $g_{3}$ value, which now differs only 100 ppm from the experimental value. GIAO-KOSEKI seems to overestimate this value slightly, as has been noted before in similar cases. ${ }^{47}$ Nevertheless, the differences between the methods are very small and, considering the computational cost of the GIAOFULL calculation, an effective one-electron treatment of spinorbit coupling (KOSEKI or AMFI) would be most efficient. By selecting a CG approach, the computational cost can be reduced even further, but the common gauge has to be chosen carefully as it influences the calculated $g$ tensor considerably.
Table 3 Gauge dependence of the calculated $g$ tensor in the CG-AMFI scheme of MAG/ReSpect. $\Delta g_{1-3}$ values (in ppm) and angle deviations $\Psi_{1-3}$ for the principal directions (in ${ }^{\circ}$ ) are given with respect to the GIAO-FULL scheme. COM = Center Of Mass

\begin{tabular}{lrrrrrr}
\hline Gauge origin & \multicolumn{1}{c}{$\Delta g_{1}$} & \multicolumn{1}{c}{$\Delta g_{2}$} & $\Delta g_{3}$ & $\Psi_{1}$ & $\Psi_{2}$ & $\Psi_{3}$ \\
\hline COM & 89 & 66 & 34 & 3 & 10 & 10 \\
C1 & 73 & 65 & -24 & 1 & 3 & 3 \\
C2 & 17 & -149 & -10 & 1 & 3 & 3 \\
N3 & 45 & -185 & 58 & 4 & 11 & 10 \\
O4 & 148 & 174 & 33 & 2 & 19 & 19 \\
O5 & 42 & 83 & -38 & 1 & 14 & 14 \\
H6 & -1 & -344 & 61 & 4 & 9 & 9 \\
H7 & 93 & -183 & 94 & 4 & 17 & 16 \\
H8 & 66 & -63 & 94 & 5 & 13 & 12 \\
H9 & -48 & -292 & -31 & 0 & 2 & 2 \\
\hline
\end{tabular}

Table 3 lists how CG-AMFI deviates from the GIAO-FULL approach for various choices of the common gauge. Differences of up to $300 \mathrm{ppm}$ for the $g$ values and $20^{\circ}$ for the principal directions occur, comparable to the gauge effects observed in another study on $N$-acetylglycine. ${ }^{29}$ A common gauge at the position of the $\mathrm{C} 1$ atom yields the best correspondence with the GIAO-FULL methodology and was therefore selected for all other CG-AMFI calculations.

The GIAO-KOSEKI method of Gaussian03 was used to examine to what extent the density functional and basis set affect the calculated $g$ tensor, summarized in Table 4. The use of a hybrid functional in the cluster calculations seems to be a prerequisite for a good match with experiment. In fact, the

Table 4 Density functional and basis set dependence of $g$-tensor calculations in a cluster approach, using the GIAO-KOSEKI methodology. A TZVP basis set was used in part (a) and the B3LYP functional in part (b)

\begin{tabular}{llll}
\hline & $g_{\text {iso }}$ & $g_{\text {aniso }}$ & Angle \\
\hline (a) Density functional & & & \\
B3LYP & 2.0036 & 2.0022 & 18 \\
& & 2.0040 & 16 \\
B3PW91 & 2.0035 & 2.0045 & 13 \\
& & 2.0022 & 18 \\
PBE0 & & 2.0039 & 16 \\
& 2.0035 & 2.0045 & 12 \\
BP86 & & 2.0022 & 18 \\
& & 2.0039 & 16 \\
PW91 & 2.0059 & 2.0044 & 13 \\
& & 2.0043 & 60 \\
(b) Basis set & & 2.0046 & 89 \\
EPR-II & 2.0059 & 2.0089 & 76 \\
& & 2.0043 & 59 \\
TZVP & & 2.0046 & 89 \\
& 2.0035 & 2.0089 & 76 \\
TZVP-SV & & 2.0022 & 18 \\
& & 2.0039 & 15 \\
DZVP & 2.0036 & 2.0044 & 12 \\
& & 2.0022 & 18 \\
DZVP-SV & 2.0036 & 2.0040 & 16 \\
& & 2.0045 & 13 \\
& & 2.0023 & 18 \\
& 2.0036 & 2.0040 & 16 \\
& & 2.0045 & 12 \\
& & 2.0022 & 17 \\
& & 2.0040 & 15 \\
& & 2.0045 & 12 \\
& & 2.0022 & 17 \\
& & 2.0040 & 14 \\
& & 2.0045 & 11 \\
\hline
\end{tabular}


B3LYP, B3PW91 $45,62-64$ and PBE0 ${ }^{65,66} \mathrm{~g}$ tensors hardly differ. Pure GGA functionals, such as BP86 $6^{33,67}$ and PW91, ${ }^{62-64}$ also show a remarkably similar pattern, but this time the calculations are completely off. Not only are the principal values significantly different from their experimental counterparts (with errors of up to $4000 \mathrm{ppm}$ ), the principal directions are entirely misaligned. It is intriguing that GGA functionals completely fail in the cluster calculations, whereas they produce a good match with experiment in the periodic all-electron calculations. This failure is a clear boundary effect, due to the limited size of the cluster. In the BP86 and PW91 cluster calculations, part of the spin density resides on atoms at the edges of the cluster, reducing the spin density on the C2 carbon to about $70 \%$ (instead of roughly $90 \%$ in the hybrid cluster calculations and the periodic $\mathrm{CP} 2 \mathrm{~K}$ calculation). In other computational studies using a cluster approach on similar glycinederived radicals ${ }^{29,30}$ no such boundary effects were observed. There, neither the radical nor its surrounding molecules were zwitterionic, as is the case in solid-state glycine. Most likely, it is this dipolar character of the constituting molecules that makes the cluster approach in this case more prone to boundary effects.

The basis set does not seem to have a significant impact on the accuracy of the $g$ tensor calculation, as has been noted before. ${ }^{47}$ Tailor-made basis sets for the calculation of EPR properties, such as EPR-II, ${ }^{68}$ offer only a slight improvement over TZVP for the principal values (of the order of $100 \mathrm{ppm}$ ) and give virtually the same result for the principal directions. Even less extended sets of basis functions succeed in reproducing the experimental $g$ tensor: the DZVP basis set, ${ }^{42}$ or a combination of TZVP or DZVP for the atoms of the radical and the Dunning-Hay SV basis ${ }^{69}$ for all other atoms in the cluster (marked TZVP-SV or DZVP-SV).

\section{Cluster calculations_-cluster size effect}

Recently, Barone and Causà ${ }^{28}$ reported that a larger 8-Clustercontaining two glycine molecules in addition to the 6-Cluster-would yield $g$ values for the glycine radical that are in better accordance with the experiment of Brustolon et al. ${ }^{20}$ Unfortunately, they misinterpreted the experimental results and compared their calculated isotropic $g$ values with the non-diagonal $g$ value measured along the crystallographic $\langle c\rangle$ axis. Comparing their calculated principal values - (2.0048, $2.0041,2.0028)$ for the 8 -Cluster, $(2.0042,2.0037,2.0022)$ for the 6-Cluster-with the experimental data of Collins and Whiffen, ${ }^{21}$ it turns out that the former actually leads to an overall worse agreement with experiment. The somewhat counterintuitive result that a bigger cluster-and therefore a more extended incorporation of the molecular environment of the radical-performs worse prompted us to closer examine the effect of the cluster size on the calculated EPR properties.

The 8-Cluster was therefore gradually reduced by iteratively eliminating the glycine molecule that is most distant from the central radical. This procedure resulted in 9 clusters, which are shown in Fig. 2-the simplest 'cluster' contains only the central glycine radical. The results of $g$ - and hyperfine tensor calculations (using GIAO-KOSEKI) for these structures are presented in Table 5-g-tensor calculations with the CG-AMFI methodology yielded comparable results. They clearly point out that the $g$ tensor is - more than anything elsesusceptible to the cluster size. Good overall correspondence with the experimental $g$ tensor is only found for the 5-Cluster, 6-Cluster and 7-Cluster. The other clusters yield principal values that are often much too high (e.g. 2.0108), accompanied by unacceptable deviations between the theoretical and measured principal directions (with angle values of $70^{\circ}-80^{\circ}$ ). Especially the $g_{2}$ and $g_{3}$ principal values and directions are very responsive to changes in the cluster constitution $-g_{1}$ is usually correctly predicted (except in the 4-Cluster). In other computational studies on $\pi$-type organic radicals, ${ }^{14,70}$ the $g_{2}$ and $g_{3}$ values have similarly been found to be quite susceptible to changes in the molecular environment.

The proton hyperfine coupling tensors are much less sensitive to these variations. As soon as the two glycine molecules adjacent to the radical amino group are taken into account

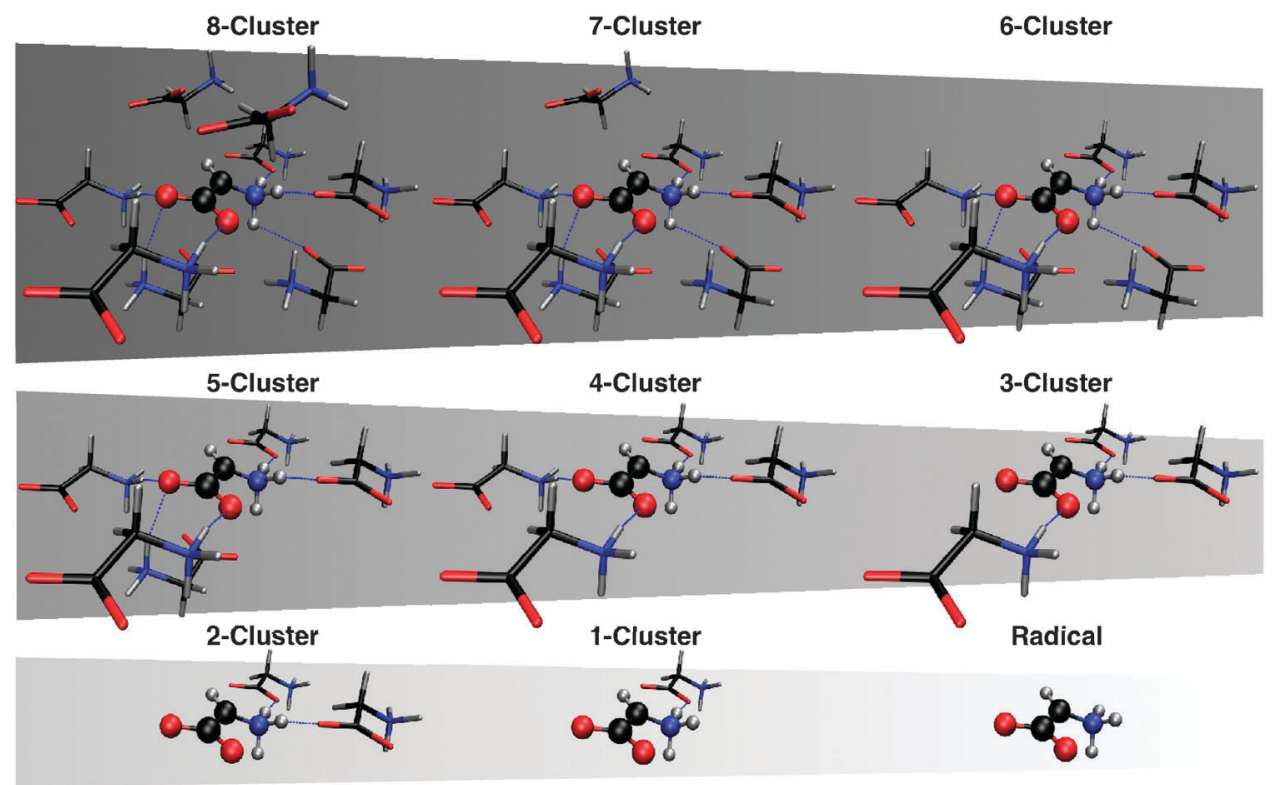

Fig. 2 Structures of 9 clusters containing the glycine radical (ball-and-stick, central) and several undamaged glycine molecules. 
Table 5 Effect of the cluster size on calculated $g$ - and hyperfine tensors. Isotropic $\left(A_{\text {iso }}\right)$ and anisotropic $\left(A_{\text {aniso }}\right)$ hyperfine coupling values are in MHz. All angles (in degrees) indicate the deviation between corresponding calculated and experimental principal directions. At the bottom, the experimental data from ref. 21 are reproduced. The GIAO-KOSEKI method was used, together with a TZVP basis set and the B3LYP functional

\begin{tabular}{|c|c|c|c|c|c|c|c|c|c|c|c|c|c|c|c|c|}
\hline & \multicolumn{3}{|c|}{$g$ tensor } & \multicolumn{13}{|c|}{ Proton hyperfine coupling tensors } \\
\hline & $g_{\text {iso }}$ & $g_{\text {aniso }}$ & Angle & H6 & & & H7 & & & $\mathrm{H} 8$ & & & H9 & & & \\
\hline \multirow[t]{3}{*}{ Radical } & 2.0058 & 2.0023 & 16 & 3.7 & & & 83.1 & & & 88.5 & & & -54.5 & & & $A_{\text {iso }}$ \\
\hline & & 2.0046 & 77 & -5.0 & -3.7 & 8.7 & -4.6 & -3.9 & 8.5 & -6.0 & -3.8 & 9.8 & -32.4 & -2.5 & 34.9 & $A_{\text {aniso }}$ \\
\hline & & 2.0104 & 75 & 13 & 12 & 11 & 31 & 32 & 11 & 19 & 20 & 7 & 4 & 10 & 9 & Angle \\
\hline \multirow[t]{3}{*}{ 1-Cluster } & 2.0048 & 2.0022 & 18 & 2.8 & & & 84.4 & & & 93.0 & & & -57.1 & & & $A_{\text {iso }}$ \\
\hline & & 2.0045 & 75 & -5.1 & -3.8 & 8.9 & -4.9 & -3.9 & 8.8 & -5.8 & -4.2 & 10.1 & -33.9 & -2.3 & 36.3 & $A_{\text {aniso }}$ \\
\hline & & 2.0076 & 72 & 13 & 12 & 11 & 27 & 28 & 11 & 21 & 22 & 8 & 4 & 11 & 9 & Angle \\
\hline \multirow[t]{3}{*}{ 2-Cluster } & 2.0046 & 2.0023 & 17 & 1.9 & & & 64.4 & & & 96.1 & & & -54.0 & & & $A_{\text {iso }}$ \\
\hline & & 2.0050 & 72 & -4.8 & -4.0 & 8.8 & -6.1 & -3.1 & 9.1 & -5.8 & -4.3 & 10.1 & -34.3 & -2.0 & 36.3 & $A_{\text {aniso }}$ \\
\hline & & 2.0066 & 69 & 7 & 9 & 11 & 4 & 4 & 2 & 8 & 9 & 7 & 5 & 11 & 10 & Angle \\
\hline \multirow[t]{3}{*}{ 3-Cluster } & 2.0042 & 2.0022 & 15 & 1.6 & & & 63.8 & & & 96.1 & & & -56.5 & & & $A_{\text {iso }}$ \\
\hline & & 2.0042 & 67 & -4.9 & -4.0 & 8.9 & -6.6 & -3.2 & 9.7 & -6.1 & -4.2 & 10.3 & -34.5 & -2.4 & 37.0 & $A_{\text {aniso }}$ \\
\hline & & 2.0062 & 65 & 6 & 9 & 10 & 2 & 2 & 3 & 4 & 5 & 6 & 5 & 11 & 10 & Angle \\
\hline \multirow[t]{3}{*}{ 4-Cluster } & 2.0066 & 2.0044 & 36 & 1.2 & & & 54.9 & & & 82.0 & & & -48.8 & & & $A_{\text {iso }}$ \\
\hline & & 2.0046 & 88 & -4.2 & -3.5 & 7.8 & -5.8 & -2.8 & 8.7 & -5.3 & -3.5 & 8.8 & -30.5 & -1.8 & 32.3 & $A_{\text {aniso }}$ \\
\hline & & 2.0108 & 85 & 6 & 10 & 10 & 4 & 5 & 4 & 6 & 6 & 5 & 5 & 9 & 8 & Angle \\
\hline \multirow[t]{3}{*}{ 5-Cluster } & 2.0036 & 2.0022 & 18 & 0.8 & & & 64.2 & & & 85.0 & & & -55.7 & & & $A_{\text {iso }}$ \\
\hline & & 2.0040 & 14 & -4.9 & -4.1 & 8.9 & -7.1 & -3.3 & 10.4 & -7.0 & -3.5 & 10.6 & -35.2 & -2.7 & 37.9 & $A_{\text {aniso }}$ \\
\hline & & 2.0047 & 12 & 4 & 10 & 10 & 12 & 12 & 5 & 3 & 3 & 2 & 5 & 11 & 9 & Angle \\
\hline \multirow[t]{3}{*}{ 6-Cluster } & 2.0036 & 2.0022 & 18 & 0.4 & & & 63.9 & & & 86.8 & & & -55.4 & & & $A_{\text {iso }}$ \\
\hline & & 2.0040 & 16 & -4.8 & -4.1 & 8.9 & -7.2 & -3.3 & 10.6 & -7.3 & -3.6 & 10.8 & -34.5 & -2.9 & 37.4 & $A_{\text {aniso }}$ \\
\hline & & 2.0045 & 12 & 5 & 11 & 10 & 11 & 12 & 6 & 4 & 5 & 4 & 5 & 10 & 9 & Angle \\
\hline \multirow[t]{3}{*}{ 7-Cluster } & 2.0036 & 2.0022 & 18 & 0.7 & & & 63.9 & & & 88.4 & & & -56.7 & & & $A_{\text {iso }}$ \\
\hline & & 2.0041 & 19 & -4.9 & -4.1 & 9.0 & -7.1 & -3.4 & 10.5 & -7.2 & -3.5 & 10.8 & -34.9 & -2.9 & 37.8 & $A_{\text {aniso }}$ \\
\hline & & 2.0045 & 14 & 3 & 10 & 10 & 11 & 11 & 5 & 5 & 5 & 4 & 5 & 9 & 8 & Angle \\
\hline \multirow[t]{3}{*}{ 8-Cluster } & 2.0060 & 2.0040 & 12 & 0.9 & & & 59.3 & & & 80.0 & & & -50.5 & & & $A_{\text {iso }}$ \\
\hline & & 2.0044 & 83 & -4.4 & -3.7 & 8.1 & -6.2 & -3.1 & 9.3 & -6.5 & -3.1 & 9.7 & -31.5 & -2.6 & 34.0 & $A_{\text {aniso }}$ \\
\hline & & 2.0096 & 82 & 4 & 10 & 10 & 12 & 12 & 4 & 6 & 7 & 4 & 5 & 10 & 8 & Angle \\
\hline \multirow[t]{3}{*}{ Exp } & 2.0036 & 2.0032 & & 3.3 & & & 62.0 & & & 82.0 & & & -63.7 & & & $A_{\text {iso }}$ \\
\hline & & 2.0035 & & -7.3 & -1.8 & 9.2 & -6.0 & -3.2 & 9.3 & -6.1 & -4.0 & 10.1 & -33.8 & 1.9 & 31.9 & $A_{\text {aniso }}$ \\
\hline & & 2.0042 & & & & & & & & & & & & & & \\
\hline
\end{tabular}

(2-Cluster), calculations yield essentially correct hyperfine tensors. The poor performance of the Radical and 1-Cluster models corroborates earlier findings that the molecular environment needs to be taken into account for an overall correct reproduction of the measured hyperfine coupling tensors. ${ }^{16}$ The higher susceptibility of the $g$-tensor calculation to the cluster size reflects the rather global character of this spectroscopic property, indicative of the total molecular system under consideration. The hyperfine tensors, on the other hand, are local indicators, only to some extent dependent on the (closest) molecular environment.

Although we were unable to exactly reproduce the 8-Cluster results of ref. 28 (owing to differences in the structure), our calculations also indicate that this cluster performs (much) worse than the 6-Cluster in the calculation of the $g$ tensor. In another theoretical study, on the sulfur-centered radical in the thiocytosine crystal, a similar poor performance of a bigger cluster model was also reported. ${ }^{71}$ These authors attributed the variability of the $g$ tensor to geometrical relaxation issues, since they used several cluster approaches to arrive at an optimized structure for the radical and its molecular environment. Our calculations on the glycine radical point out that it is in fact much worse: the mere composition of a cluster can be determining for the quality of the $g$-tensor calculation.

\section{Analysis of atomic contributions to the $g$ tensor}

If effective atomic spin-orbit operators are used (as is the case in KOSEKI or AMFI), MAG/ReSpect allows the breakdown of the dominant spin-orbit contribution to the total $g$ tensor (eqn (1)) in atomic contributions. This offers unique insight in the calculated $g$ tensor and also why it is so sensitive to the cluster composition. In Table 6, such an atomic analysis is performed for the CG-AMFI $g$ tensor of the Radical and 6-Cluster models, determined using the B3LYP functional, TZVP basis and $\mathrm{C} 1$ atom as a common gauge. In both cases, the $g_{2}$ and $g_{3}$ values of the total $g$ tensor $\left(\Delta g_{\text {total }}\right)$ are almost entirely determined by the spin-orbit part $\left(\Delta g_{\mathrm{SO} / \mathrm{OZ}}\right)$, which in turn heavily depends on the contributions of the five heavy atoms constituting the radical. In the 6-Cluster, these atoms together account for $1433 \mathrm{ppm}$ and $1878 \mathrm{ppm}$ of the $\Delta g_{2}$ and

Table 6 Analysis of the total $g$ tensor $\left(\Delta g_{\text {total }}\right)$ in terms of spin-orbit contributions: $\Delta g_{\mathrm{SO} / \mathrm{OZ}}$ indicates the entire spin-orbit/orbital-Zeeman part and is further broken down into contributions due to the heavy atoms constituting the radical ( $\mathrm{C} 1$ to $\mathrm{N} 5)$. All $g$ values are given in $\mathrm{ppm}$, relative to the free electron value. $\rho$ indicates the Mulliken spin density on a particular atom

\begin{tabular}{|c|c|c|c|c|c|c|c|c|}
\hline & \multicolumn{4}{|c|}{ 6-Cluster } & \multicolumn{4}{|c|}{ Radical } \\
\hline & $\Delta g_{1}$ & $\Delta g_{2}$ & $\Delta g_{3}$ & $\rho$ & $\Delta g_{1}$ & $\Delta g_{2}$ & $\Delta g_{3}$ & $\rho$ \\
\hline$\Delta g_{\text {total }}$ & 6 & 1620 & 1992 & & 12 & 2023 & 7343 & \\
\hline$\Delta g_{\mathrm{SO} / \mathrm{OZ}}$ & 32 & 1572 & 2010 & & 87 & 1998 & 7135 & \\
\hline $\mathrm{Cl}$ & -1 & -49 & 55 & -0.04 & 0 & 40 & -8 & -0.10 \\
\hline $\mathrm{C} 2$ & 21 & 170 & 313 & 0.91 & 6 & 71 & 75 & 0.86 \\
\hline N3 & -3 & 35 & 229 & -0.04 & -12 & 32 & -31 & -0.04 \\
\hline $\mathrm{O} 4$ & -10 & 445 & 662 & 0.07 & 62 & 568 & 482 & 0.03 \\
\hline $\mathrm{O} 5$ & -8 & 831 & 620 & 0.08 & 76 & 1402 & 7099 & 0.23 \\
\hline
\end{tabular}


$\Delta g_{3}$ values, respectively. Since the radical hydrogens barely contribute (data not shown), the remaining 139 and $132 \mathrm{ppm}$ of the spin-orbit contributions to $g_{2}$ and $g_{3}$, respectively, are determined by the environment. The effect of the environment on the $g$ tensor is therefore not a direct one: it hardly contributes to the $g_{2}$ and $g_{3}$ values.

Further examining the individual atomic contributions, it is clear that $\mathrm{C} 1$ overall contributes the least to the size of the $g$ values. Because this atom is also in the middle of the other heavy atoms of the radical, this might provide a rationale for the performance of the CG-AMFI method with the common gauge origin set at this point (see above). The radical carbonyl oxygens largely control the size of the $g_{2}$ and $g_{3}$ values, in accordance with a previous study on $\mathrm{N}$-acetylglycine. ${ }^{29}$ In the 6-Cluster model, both oxygen contributions are roughly of the same order of magnitude. This is obviously not the case in the Radical model, where $\mathrm{O} 5$ dominates all other contributions, effectively resulting in poor correspondence with the experimental $g$ tensor. The huge spin-orbit contribution for this atom follows the increased Mulliken spin density at this position (also reported in Table 6). In the cluster calculation, the molecular environment causes the spin density to distribute differently over the heavy atoms of the radical, with more emphasis on C2 (0.91). In this way, the molecular environment in the cluster model indirectly determines the $g$ tensor. But an ill-composed cluster can shift the delicately balanced spin density distribution on the central radical moiety. This is exactly the case in, e.g. the 4-Cluster and 8-Cluster models. Just as in the isolated radical, the spin density is shifted away from $\mathrm{C} 2$, giving rise to a $g$ tensor that is in poor agreement with experiment.

The atomic analysis points out that the molecular environment in a cluster model of the solid-state glycine radical does not contribute directly to the $g_{2}$ and $g_{3}$ values, which are largely determined by the central radical moiety. Instead, the environment polarizes the spin density on this radical, indirectly influencing the $g$ tensor. That polarizing effect is largely electrostatic in nature. Recalculating the $g$-tensor for the 8-Cluster model in which the two additional molecules (with respect to the 6-Cluster) are replaced by point charges ${ }^{36}$ yields comparably poor results: the principal values are $2.0035,2.0044$, and 2.0078.

In previous works on similar glycyl radicals, ${ }^{29,30}$ the composition of a supermolecular cluster model proved to have a far less dramatic influence on the $g_{2}$ and $g_{3}$ values of the calculated $g$ tensor. But there, the spin density was more distributed over a mostly planar radical structure in which much smaller density shifts occurred as a function of the cluster composition. In our glycine system, it is well established that the central radical is not planar. ${ }^{17}$ Therefore the spin density is more concentrated, making it more susceptible to alterations of the molecular environment, especially when that environment is made up of highly polarized zwitterionic glycine molecules.

\section{Temperature dependence}

To go beyond the static nature of the previous results, $g$-tensor calculations were performed on 400 structural snapshots from a 2.4 ps trajectory of an MD simulation at $300 \mathrm{~K}$. Using both GIAO-KOSEKI and CG-AMFI (with atom $\mathrm{Cl}$ as a common gauge), the 6-Cluster model was selected for these snapshots, in combination with the B3LYP density functiona ${ }^{45}$ and a DZVP basis. ${ }^{42}$ As argued above, this level of theory is able to correctly capture the electronic structure of the glycine radical at an affordable computational cost. By averaging over all snapshots:

$$
\langle\mathbf{g}\rangle=\frac{1}{N} \sum_{i}^{N=400} \mathbf{g}_{i}
$$

an average $g$ tensor at $300 \mathrm{~K}$ was obtained, which was subsequently diagonalized:

$$
\left(\begin{array}{lll}
\mathbf{V}_{\mathbf{1}} & \mathbf{V}_{\mathbf{2}} & \mathbf{V}_{\mathbf{3}}
\end{array}\right)^{\mathbf{T}}\langle\mathbf{g}\rangle\left(\begin{array}{lll}
\mathbf{V}_{\mathbf{1}} & \mathbf{V}_{\mathbf{2}} & \mathbf{V}_{\mathbf{3}}
\end{array}\right)=\left[\begin{array}{lll}
g_{1} & & \\
& g_{2} & \\
& & g_{3}
\end{array}\right]
$$

This yields three average eigenvalues $g_{1}$ to $g_{3}$ (expectation values) and their corresponding principal directions $\mathbf{V}_{\mathbf{1}}$ to $\mathbf{V}_{\mathbf{3}}$ represented by $3 \times 1$ column matrices.

The correspondence of these average $g$ tensors at $300 \mathrm{~K}$ with experiment is shown in Table 7 for both the GIAO-KOSEKI and CG-AMFI implementation. Comparing with the static results, the dynamics offer no real improvement. The $g_{1}$ value has slightly increased but the $g_{2}$ and $g_{3}$ principal values are more alike, seemingly converging towards one average value: the dynamics have rendered the $g$ tensor more axial. The origin of this axial character can be traced by examining the projections of the calculated $g$ tensors at all snapshots $\left(\mathbf{g}_{i}\right)$ along the above-mentioned, average principal directions $\mathbf{V}_{1}-\mathbf{V}_{3}$ :

$$
g_{i}^{\text {proj- } \alpha}=\left\|\mathbf{g}_{i} \mathbf{V}_{\alpha}\right\|
$$

with $\alpha=1-3$. The resulting 400 projected values for $g^{\text {proj-1 }}, g^{\text {proj-2 }}$ and $g^{\text {proj-3 }}$ are shown as histograms in Fig. 3. The distribution for $g_{1}$ is rather narrow, but those for $g_{2}$ and $g_{3}$ are broad and overlap with each other. Clearly, these principal values are most susceptible to the structural and associated electronic changes during the dynamics, complicating a clear distinction between them. The same effect is apparent for the principal directions of the average $g$ tensor at $300 \mathrm{~K}$. Whereas $\mathbf{V}_{\mathbf{1}}$ lies within the $20^{\circ}$ mark from the experimental principal direction - very much comparable to the static results $-\mathbf{V}_{\mathbf{2}}$ and $\mathbf{V}_{\mathbf{3}}$ deviate substantially: $30-40^{\circ}$. Here also, the distinction between the latter two principal directions has

Table 7 Average $g$ tensors obtained from sampled snapshots along a $300 \mathrm{~K}$ molecular dynamics simulation compared to the results of a static calculation. The B3LYP functional was used in combination with a DZVP basis set. The $\mathrm{Cl}$ atom was used as a common gauge in the CG-AMFI calculation

\begin{tabular}{lllll}
\hline Implementation & & $g_{\text {iso }}$ & $g_{\text {aniso }}$ & Angle \\
\hline GIAO-KOSEKI & Static & 2.0036 & 2.0022 & 17 \\
& & & 2.0040 & 15 \\
& & & 2.0045 & 12 \\
& Dynamic & 2.0037 & 2.0024 & 16 \\
& & & 2.0042 & 29 \\
CG-AMFI & Static & \multirow{2}{*}{2.0035} & 2.0044 & 28 \\
& & & 2.0023 & 18 \\
& & & 2.0039 & 15 \\
& Dynamic & 2.0036 & 2.0025 & 12 \\
& & & 2.0041 & 32 \\
& & & 2.0042 & 30 \\
\hline
\end{tabular}



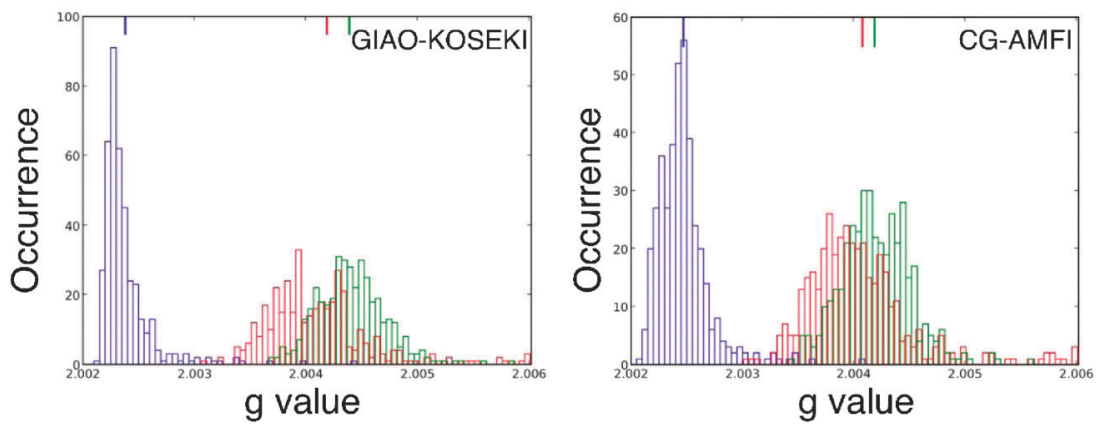

Fig. 3 Histograms of the 400 projected $g$-tensor values, calculated using the GIAO-KOSEKI and CG-AMFI implementations. Blue, red and green boxes, respectively, indicate the occurrence of the $g^{\text {proj-1 }}, g^{\text {proj-2 }}$ and $g^{\text {proj-3 }}$ values as resulting from projection along the averaged $\mathbf{V}_{\mathbf{1}}-\mathbf{V}_{\mathbf{3}}$ principal directions (see eqn (5)-(7)). For each distribution, average values (corresponding to the principal values in Table 4) are shown at the top of the plots.

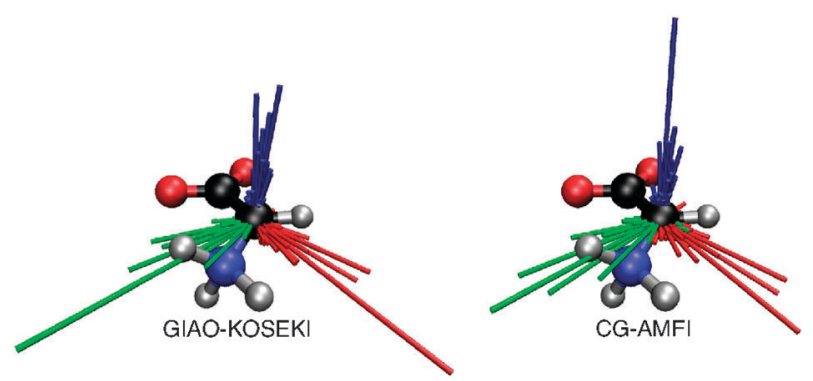

Fig. 4 Distribution of principal directions (blue: $g_{1}$, red: $g_{2}$, green: $g_{3}$ principal direction) for the 400 snapshots from the MD simulation. Each bar is oriented along the center of a bin on a $10 \times 10 \times 103 \mathrm{D}$ grid, spanning all principal directions. The size of the bar indicates the population in that bin.

become confused. Though the average principal directions $\mathbf{V}_{\mathbf{1}}-\mathbf{V}_{\mathbf{3}}$ (which are strictly defined by eqn (5) and (6)) do not correspond to the averages of the $3 \times 400$ principal directions obtained throughout the dynamics, it still is informative to examine the variation of the latter. This is graphically represented in Fig. 4 . All $3 \times 400$ principal directions were mapped on a $3 \mathrm{D}$ grid of $10 \times 10 \times 10$ bins. Each bar in Fig. 4 is oriented along the center of one of these bins, its size indicating the population. Clearly, the distribution for the $g_{1}$ principal direction (in blue) is narrower than those for the $g_{2}$ and $g_{3}$ directions (green and red, respectively), the latter two almost overlapping with each other.

\section{Conclusions}

In this work, we examined the $g$ tensor of the main ${ }^{+} \mathrm{NH}_{3}-{ }^{\bullet} \mathrm{CH}-\mathrm{COO}^{-}$radiation-induced radical in solid-state $\alpha$-glycine, relying on both periodic and cluster DFT calculations. Even though the methodology for $g$-tensor calculations is well established, its application to this specific system is quite challenging. Especially the principal values and -directions associated with the intermediate and major principal $g$-tensor components $\left(g_{2}\right.$ and $\left.g_{3}\right)$ are most susceptible to the adopted methodology. In a periodic approach, only the all-electron GAPW implementation of the $g$ tensor in $\mathrm{CP} 2 \mathrm{~K}$ yields a favorable agreement with the experiment. In a cluster approach, the size of the basis set and the particular nature of the $g$-tensor implementation are only of secondary importance for the final result of the $g$-tensor calculation. Instead, the use of a hybrid functional and the selection of a suitable cluster size seem more essential in the present case. The reduced computational cost of such cluster calculations makes them particularly attractive as compared to periodic calculations, although both schemes have complementary strengths and weaknesses.

The selection of the cluster size is perhaps the most important computational parameter in the cluster calculation of the $g$ tensor. This spectroscopic property is a global indicator of the total molecular system under consideration, in contrast to the hyperfine tensors, which are much more local. As such, the calculated $g$ tensor varies significantly with increasing cluster size, yielding only a good agreement with experiment when 5-7 glycine molecules in the immediate environment of the central glycine radical are incorporated in the cluster model. Further expansion of the cluster size is not straightforward and can even lead to a worse agreement with experiment: a cluster containing 8 glycine molecules in addition to the radical yields a $g$ tensor that is completely incompatible with the experimental data. This suggests that a cluster can effectively become too big. The glycine $g$-tensor is largely controlled by the spin-orbit contributions from the carbonyl oxygens, and by a delicately balanced spin density distribution on the radical moiety. An ill-composed cluster can shift this balance, yielding an essentially incorrect description of the radical in the condensed phase.

Finally, incorporation of temperature effects in the calculation of the $g$ tensor, with the aid of molecular dynamics simulations, does not necessarily offer a better agreement with experiment than a static calculation. Especially the $g_{2}$ and $g_{3}$ principal values and associated principal directions easily mix, yielding an average $g$ tensor that is more axial. Still, these simulations allow valuable insight into the variability and susceptibility of the $g$-tensor components to the structural and associated electronic changes during the dynamics.

\section{Acknowledgements}

This work is supported by the Fund for Scientific Research-Flanders (FWO), the Research Board of the Ghent University and BELSPO in the frame of IAP 6/27. Part of the computational resources and services used in this work were provided by Ghent University. We gratefully acknowledge Dr Hendrik De Cooman of the Ghent University for his 
reanalysis of the experimental EPR and ENDOR angular variations. Work at TU Berlin has been supported within the framework of the DFG excellence cluster on "Unifying Concepts in Catalysis".

\section{References}

1 J. Stubbe and W. A. van der Donk, Chem. Rev., 1998, 98, 705-762.

2 A. Mozumder and Y. Hatano, Charged particle and photon interactions with matter: chemical, physicochemical and biological consequences with applications, Dekker, New York, NY, 2004.

3 A. Adhikary, A. Kumar and M. D. Sevilla, Radiat. Res., 2006, 165, $479-484$.

4 N. Jayatilaka and W. H. Nelson, J. Phys. Chem. B, 2007, 111, $800-810$.

5 H. De Cooman, G. Vanhaelewyn, E. Pauwels, E. Sagstuen, M. Waroquier and F. Callens, J. Phys. Chem. B, 2008, 112, 15045-15053.

6 A. Krivokapic, J. N. Herak and E. Sagstuen, J. Phys. Chem. A, 2008, 112, 3597-3606.

7 E. Pauwels, H. De Cooman, G. Vanhaelewyn, E. Sagstuen, F. Callens and M. Waroquier, J. Phys. Chem. B, 2008, 112, 15054-15063.

8 K. K. Sharma, Y. Razskazovskiy, S. Purkayastha and W. A. Bernhard, J. Phys. Chem. B, 2009, 113, 8183-8191.

9 D. M. Close, J. Phys. Chem. A, 2010, 114, 1860-1867.

10 M. Kaupp, M. Bühl and V. G. Malkin, Calculation of NMR and EPR parameters: theory and applications, Wiley- $\mathrm{VCH}$, Weinheim, 2004.

11 A. Krivokapic, K. T. Ohman, M. Munthe, W. H. Nelson, E. O. Hole and E. Sagstuen, Radiat. Res, 2010, 173, 689-702.

12 Y. Y. Zhou and W. H. Nelson, J. Phys. Chem. B, 2010, 114, $5567-5582$.

13 H. De Cooman, E. Pauwels, H. Vrielinck, E. Sagstuen, M. Waroquier and F. Callens, J. Phys. Chem. B, 2010, 114, 666-674.

14 J. R. Asher, N. L. Doltsinis and M. Kaupp, Magn. Reson. Chem., 2005, 43, S237-S247.

15 E. Pauwels, T. Verstraelen and M. Waroquier, Spectrochim. Acta, Part A, 2008, 69, 1388-1394.

16 E. Pauwels, V. Van Speybroeck and M. Waroquier, J. Phys. Chem. A, 2004, 108, 11321-11332.

17 E. Pauwels, T. Verstraelen, H. De Cooman, V. Van Speybroeck and M. Waroquier, J. Phys. Chem. B, 2008, 112, 7618-7630.

18 E. F. Garman, Acta Crystallogr., Sect. D: Biol. Crystallogr., 2010, 66, 339-351.

19 H. C. Box, H. G. Freund and E. E. Budzinski, J. Am. Chem. Soc, 1966, 88, 658-661.

20 M. Brustolon, V. Chis, A. L. Maniero and L. C. Brunel, J. Phys. Chem. A, 1997, 101, 4887-4892.

21 M. A. Collins and D. H. Whiffen, Mol. Phys., 1966, 10, 317-325.

22 J. R. Morton, J. Am. Chem. Soc., 1964, 86, 2325-2329.

23 E. Sagstuen, A. Sanderud and E. O. Hole, Radiat. Res., 2004, 162, $112-119$.

24 A. Sanderud and E. Sagstuen, J. Phys. Chem. B, 1998, 102, 9353-9361.

25 R. G. Wilks, J. B. MacNaughton, H. B. Kraatz, T. Regier, R. I. R. Blyth and A. Moewes, J. Phys. Chem. A, 2009, 113, 5360-5366.

26 F. Q. Ban, J. W. Gauld and R. J. Boyd, J. Phys. Chem. A, 2000, 104, 5080-5086.

27 V. Barone, C. Adamo, A. Grand and R. Subra, Chem. Phys. Lett., 1995, 242, 351-354.

28 V. Barone and M. Causà, Chem. Phys. Lett., 2008, 452, 89-93.

29 S. Kacprzak, R. Reviakine and M. Kaupp, J. Phys. Chem. B, 2007, 111, 811-819.

30 S. Kacprzak, R. Reviakine and M. Kaupp, J. Phys. Chem. B, 2007, 111, 820-831.

31 R. Destro, P. Roversi, M. Barzaghi and R. E. Marsh, J. Phys. Chem. A, 2000, 104, 1047-1054

32 CPMD, CPMD V3.11, Copyright IBM Corp 1990-2006. Copyright MPI fuer Festkoerperforschung Stuttgart 1997-2001.

33 J. P. Perdew, Phys. Rev. B: Condens. Matter Mater. Phys., 1986, 33, $8822-8824$.
34 A. D. Becke, J. Chem. Phys., 1992, 96, 2155-2160.

35 D. Vanderbilt, Phys. Rev. B: Condens. Matter Mater. Phys., 1990, 41, 7892-7895.

36 U. C. Singh and P. A. Kollman, J. Comput. Chem., 1984, 5, 129-145.

37 R. Car and M. Parrinello, Phys. Rev. Lett., 1985, 55, 2471-2474.

38 S. Nosé, J. Chem. Phys., 1984, 81, 511-519.

39 CP2K, CP2K, http://cp2k.berlios.de edn.

40 N. Troullier and J. L. Martins, Phys. Rev. B: Condens. Matter Mater. Phys., 1991, 43, 1993-2006.

41 M. Krack and M. Parrinello, Phys. Chem. Chem. Phys., 2000, 2, 2105-2112.

42 N. Godbout, D. R. Salahub, J. Andzelm and E. Wimmer, Can. J. Chem., 1992, 70, 560-571.

43 J. P. Perdew, K. Burke and M. Ernzerhof, Phys. Rev. Lett., 1996, 77, 3865-3868.

44 G. W. T. M. J. Frisch, H. B. Schlegel, G. E. Scuseria, M. A. Robb, J. R. Cheeseman, J. A. Montgomery, Jr., T. Vreven, K. N. Kudin, et al., Gaussian 03, Gaussian, Inc., Wallingford, CT, B.03 edn, 2004.

45 A. D. Becke, J. Chem. Phys., 1993, 98, 5648-5652.

46 V. G. Malkin, O. L. Malkina, R. Reviakine, A. V. Arbuznikov, M. Kaupp, B. Schimmelpfennig, I. Malkin, T. Helgaker and K. Ruud, MAG-ReSpect, MAG-ReSpect 1.2 edn., 2003.

47 M. Kaupp, Ab initio and Density Functional Calculations of Electronic g-Tensors for Organic Radicals, Kluwer, Dordrecht, 2003.

48 F. Neese, J. Chem. Phys., 2001, 115, 11080-11096.

49 S. Koseki, M. W. Schmidt and M. S. Gordon, J. Phys. Chem., 1992, 96, 10768-10772.

50 R. Ditchfield, Mol. Phys., 1974, 27, 789-807.

51 K. Wolinski, J. F. Hinton and P. Pulay, J. Am. Chem. Soc., 1990, 112, 8251-8260.

52 R. Declerck, V. Van Speybroeck and M. Waroquier, Phys. Rev. B. Condens. Matter Mater. Phys., 2006, 73, 115113.

53 V. Weber, M. Iannuzzi, S. Giani, J. Hutter, R. Declerck and M. Waroquier, J. Chem. Phys., 2009, 131, 014106.

54 G. Schreckenbach and T. Ziegler, J. Phys. Chem. A, 1997, 101, 3388-3399.

55 C. J. Pickard and F. Mauri, Phys. Rev. Lett, 2002, 88, 086403.

56 T. A. Keith and R. F. W. Bader, Chem. Phys. Lett., 1993, 210, 223-231.

57 M. Kaupp, R. Reviakine, O. L. Malkina, A. Arbuznikov, B. Schimmelpfennig and V. G. Malkin, J. Comput. Chem., 2002, 23, 794-803.

58 B. A. Hess, C. M. Marian, U. Wahlgren and O. Gropen, Chem. Phys. Lett., 1996, 251, 365-371.

59 B. Schimmelpfennig, Atomic Spin-Orbit Mean-Field Integral Program, Stockholms Universitet, Sweden, 1996.

60 E. Sagstuen, E. O. Hole, S. R. Haugedal and W. H. Nelson, J. Phys. Chem. A, 1997, 101, 9763-9772.

61 C. Duboc-Toia, A. K. Hassan, E. Mulliez, S. Ollagnier-de Choudens, M. Fontecave, C. Leutwein and J. Heider, J. Am. Chem. Soc., 2003, 125, 38-39.

62 J. P. Perdew, J. A. Chevary, S. H. Vosko, K. A. Jackson, M. R. Pederson, D. J. Singh and C. Fiolhais, Phys. Rev. B. Condens. Matter Mater. Phys., 1992, 46, 6671-6687.

63 J. P. Perdew, J. A. Chevary, S. H. Vosko, K. A. Jackson, M. R. Pederson, D. J. Singh and C. Fiolhais, Phys. Rev. B: Condens. Matter Mater. Phys., 1993, 48, 4978-4978.

64 J. P. Perdew, M. Emzerhof and K. Burke, J. Chem. Phys., 1996, 105, 9982-9985.

65 C. Adamo and V. Barone, J. Chem. Phys., 1999, 110, 6158-6170.

66 C. Adamo, M. Cossi and V. Barone, J. Mol. Struct. (THEOCHEM), 1999, 493, 145-157.

67 A. D. Becke, Phys. Rev. A, 1988, 38, 3098-3100.

68 N. Rega, M. Cossi and V. Barone, J. Chem. Phys., 1996, 105, 11060-11067.

69 T. H. Dunning and P. J. Hay, Modern Theoretical Chemistry, Plenum, New York, 1977, vol. 3.

70 J. R. Asher, N. L. Doltsinis and M. Kaupp, J. Am. Chem. Soc., 2004, 126, 9854-9861.

71 V. Gomzi and J. N. Herak, Chem. Phys., 2007, 333, 112-118. 